\title{
Ultrasound and Photoacoustic Imaging of the Kidney: Basic Concepts and Protocols
}

\author{
Sandra Meyer, Dieter Fuchs, and Martin Meier
}

\begin{abstract}
Noninvasive, robust, and reproducible methods to image kidneys are provided by different imaging modalities. A combination of modalities (multimodality) can give better insight into structure and function and to understand the physiology of the kidney. Magnetic resonance imaging can be complemented by a multimodal imaging approach to obtain additional information or include interventional procedures. In the clinic, renal ultrasound has been essential for the diagnosis and management of kidney disease and for the guidance of invasive procedures for a long time. Adapting ultrasound to preclinical requirements and for translational research, the combination with photoacoustic imaging expands the capabilities to obtain anatomical, functional, and molecular information from animal models. This chapter describes the basic concepts of how to image kidneys using different and most appropriate modalities.

This chapter is based upon work from the COST Action PARENCHIMA, a community-driven network funded by the European Cooperation in Science and Technology (COST) program of the European Union, which aims to improve the reproducibility and standardization of renal MRI biomarkers. This introduction chapter is complemented by two separate chapters describing the experimental procedure and data analysis.
\end{abstract}

Key words Magnetic resonance imaging (MRI), Kidney, Ultrasound imaging (US), Photoacoustic imaging (PA), Multimodality, Doppler, Contrast, Animals, Rats, Mice

\section{Introduction}

Magnetic resonance imaging, while very powerful by itself, can be complemented by a multimodal imaging approach to streamline workflow processes, obtain additional information, or include interventional procedures. Ultrasound and computed tomography are modalities of primary choice in renal imaging on patients, with the disadvantage of computed tomography exposing the body to harmful irradiation. Due to its fast, cost-effective, versatile, and nonionizing characteristics, ultrasound imaging is well-established in the clinical practice, often used for first-line diagnostics and follow-ups and as a complementary method to MRI. 
In the clinic, renal ultrasound has been essential for the diagnosis and management of kidney disease and for the guidance of invasive procedure for decades. Nevertheless, new developments to advance clinical diagnostics and medical treatments are required and strongly rely on appropriate preclinical models. Adapting the ultrasound technology to match preclinical requirements for translational studies and combining it with photoacoustic imaging, expands the researchers' capabilities to obtain anatomical, functional, and molecular information from their animal models.

MRI has the advantage of superior soft-tissue contrast, which facilitates and enables the detection and characterization of renal lesions. This chapter will give an introduction to how detailed kidney images can be created, blood flow visualized and quantified, and capillary as well as renal function assessed in vivo, noninvasively, and in real time using high-frequency ultrasound.

This introduction chapter is complemented by two separate chapters describing the experimental procedure and data analysis, which are part of this book.

This chapter is part of the book Pohlmann A, Niendorf T (eds) (2020) Preclinical MRI of the Kidney-Methods and Protocols. Springer, New York.

\section{Measurement Concepts of Ultrasound and Photoacoustics}

The basic principle of ultrasound imaging relies on detecting differences in tissue density by using sound waves with frequencies above the range of human hearing - typically in the $\mathrm{MHz}$ range. Using piezoelectric elements short, harmless ultrasound pulses are sent into the body. These sound waves are partially reflected back to the transducer by different layers of tissue and the return signals (often referred to as "echo"- -hence the term "echography" which is used as a synonym to ultrasound) are recorded. Based on the time it takes for the echo to travel into the tissue and back and the strength of the signal, an image can be computed and displayed in real time. The resulting two-dimensional, grayscale image of an area of interest is referred to as Brightness Mode or B-Mode. Tissue closest to the transducer is displayed at the top and structures further away are shown below; a scale provides depth and size information in real time. With increasing density more echo is recorded resulting in a brighter signal on the screen; therefore, dense structures (e.g., skin) are represented in white and light gray tones, whereas ultrasound gel used for coupling and fluid-filled structures inside the body appear black. Most tissues and organs are displayed in a range of grayscales in between and have a characteristic appearance ("speckle pattern") that aids in their identification. B-Mode is the standard imaging mode on ultrasound systems and worked with the most because it allows for effective visualization of anatomical structures. It can be complemented by additional imaging modes to obtain functional information which are outlined in more detail in Subheading 3. 
In ultrasound, the frequency of the sound waves sent into the tissue is of importance as it determines image resolution and penetration depth. In a clinical setting, renal ultrasound scans on adult patients are normally performed with a $3-6 \mathrm{MHz}$ curved array transducer where the term "curved" describes the shape of the transducer surface. Routine measurements include determining the length of the kidney in a sagittal view, the measurement of cortex thickness and of blood flow in the renal and interlobar arteries [1].

By using an appropriate setup and ultrasound imaging system, these parameters and a variety of others can be obtained in preclinical animal models. Dedicated small-animal micro-ultrasound systems provide high spatial resolution down to $30 \mu \mathrm{m}$ by employing higher frequencies of up to $70 \mathrm{MHz}$. While early systems for small animal imaging were equipped with mechanical probes, state-ofthe-art micro-ultrasound systems are working with digital linear array (flat transducer surface) probes and are suitable for a broad range of applications [2]. In addition to providing higher resolution than clinical machines, ultrasound systems dedicated to preclinical research also:

1. Allow for controlled transducer positioning (Fig. la, c) and include a setup for image-guided injections. While clinical ultrasound and also injections are performed handheld, a stationary setup is superior in most preclinical applications.

2. Include an integrated animal handling solution for physiological monitoring and anesthesia. These are crucial components for any kind of imaging focusing on functional analysis (blood flow, perfusion, etc.) and should be controlled and recorded carefully at all times to ensure reproducibility. However, some ultrasound applications to evaluate anatomy can be performed on conscious animals when appropriately restrained or trained.

Since ultrasound relies on the detection of soundwaves, no contrast agent is required to visualize anatomical structures, $3 \mathrm{D}$ volumes, or to assess blood flow velocities. It is noteworthy that anatomical structures and volumes measured with dedicated small animal ultrasound match results acquired with dedicated small animal MRI or $\mu \mathrm{CT}$ systems [3].

Above and beyond the capabilities of micro-ultrasound to gather anatomical and functional information, photoacoustic imaging can also be applied in a preclinical setting to add molecular information. Photoacoustic imaging is a hybrid imaging modality that combines the strengths of optical and ultrasound imaging and can be used to monitor endogenous parameters such as blood content or oxygen saturation or various exogenous contrast agents.

In this imaging approach (Fig. 2), short (a few nanoseconds) pulses of near-infrared laser light are transmitted into the tissue and 


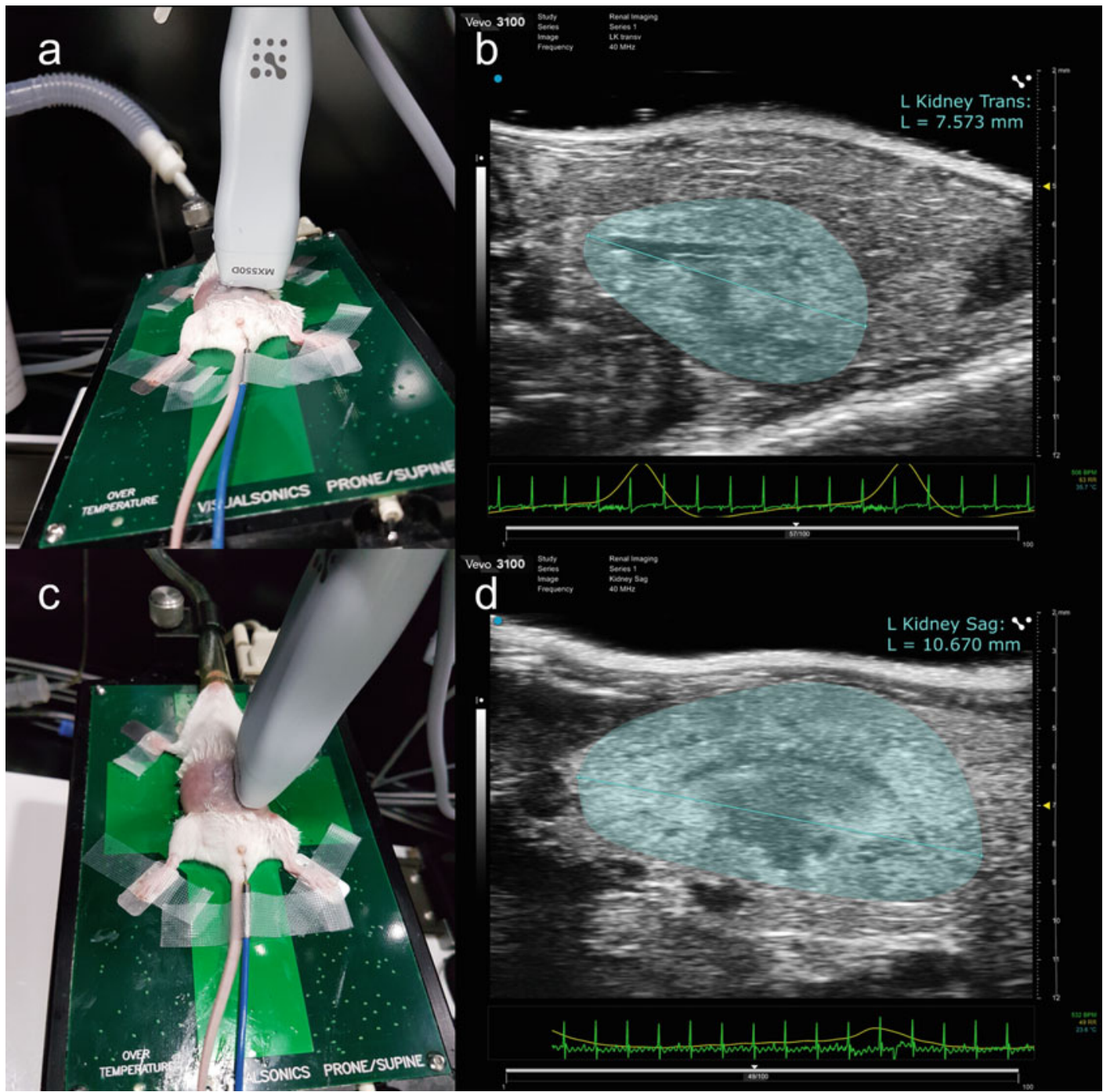

Fig. 1 Standard renal exam: animal and transducer positioning; (a) transverse position for left kidney imaging, (b) transverse US B-Mode image of the left kidney with length measurement, (c) sagittal position for left kidney imaging, (d) sagittal US B-Mode image of the left kidney with length measurement. Note the millimeter scale on the right and the physiological monitoring (ECG in green, respiration in yellow) below the US images

are selectively absorbed according to the optical properties of the tissue components. Light absorption results in a thermoelastic expansion creating a sound wave that can be localized by the ultrasound transducer and at the same resolution as the ultrasound image [4]. 


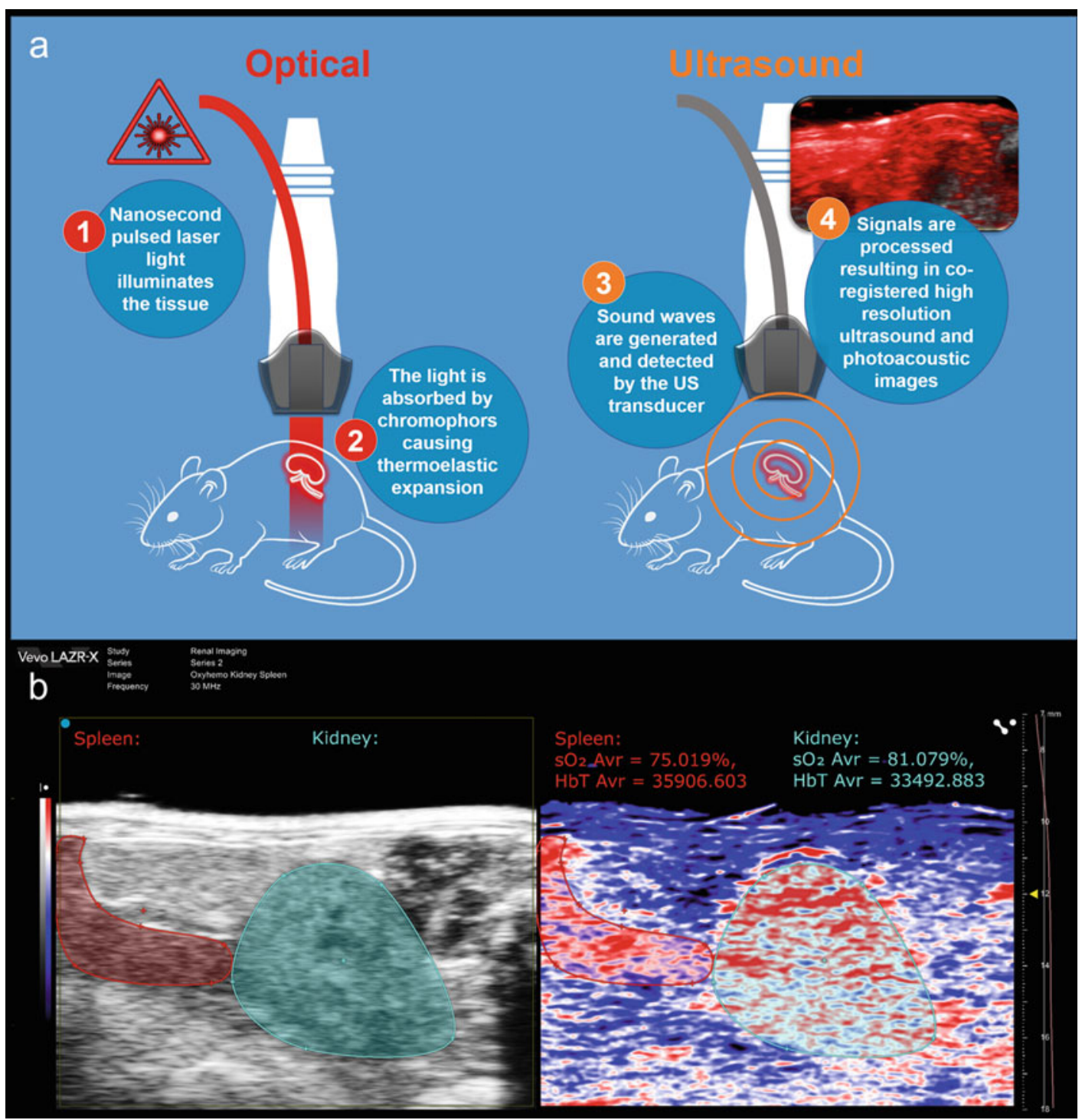

Fig. 2 Photoacoustic imaging; (a) the photoacoustic effect, (b) coregistered B-Mode image providing anatomical information (left) and photoacoustic image displaying oxygen saturation (right) in the spleen and left kidney

\section{Overview of Applications}

This section provides an overview of applications of high-frequency ultrasound and photoacoustic imaging in preclinical renal research. Examples of specific disease models and how multimodal imaging is applied are summarized in a brief review of selected publications in Table 1. 
Table 1

\section{Examples of specific disease models and how multimodal imaging is applied}

\begin{tabular}{|c|c|c|c|}
\hline Disease (animal model) & Usage of US and PA & Findings and Remarks & References \\
\hline $\begin{array}{l}\text { Polycystic kidney disease } \\
\text { (PCK rats) }\end{array}$ & $\begin{array}{l}\text { - Cyst detection } \\
\text { - Kidney and cyst volume }\end{array}$ & $\begin{array}{l}\text { Evaluation of kidneys in B-mode, power } \\
\text { Doppler, and 3D } \\
\text { - Quantification of kidney volume, } \\
\text { number of cysts and cyst volume } \\
\text { - US-based kidney volumes correlated } \\
\text { strongly with kidney weights } \\
\text { - US provided superior information in } \\
\text { comparison to microscopic analysis of } \\
\text { stained kidney sections }\end{array}$ & {$[12]$} \\
\hline $\begin{array}{l}\text { Type } 2 \text { diabetes } \\
\text { (leptin-deficient }(\mathrm{Ob} / \mathrm{Ob}) \\
\text { mice }\end{array}$ & $\begin{array}{l}\text { - Anatomical and blood } \\
\text { flow analysis } \\
\text { - Renal RI and PI; }\end{array}$ & $\begin{array}{l}\text { - RI and PI were higher in the ob/ob mice } \\
\text { (RI } 1.50 ; \text { PI } 0.81 \text { ) at } 21 \text { weeks } \\
\text { compared to lean controls (RI } 0.69 ; \text { PI } \\
1.18 \text { ) } \\
\text { - Includes echocardiography and coronary } \\
\text { flow reserve data }\end{array}$ & {$[13]$} \\
\hline $\begin{array}{l}\text { Orthotopic renal cell } \\
\text { carcinoma } \\
\text { (athymic nude mice) }\end{array}$ & - Tumor volume & $\begin{array}{l}\text { - Validation of noninvasive imaging tools } \\
\text { for monitoring of tumor growth in an } \\
\text { orthotopic renal cell carcinoma } \\
\text { xenograft } \\
\text { - Tumor volumes determined by hrUS, } \\
\mu \mathrm{CT} \text {, and MRI showed a very good } \\
\text { correlation with each other and with } \\
\text { caliper measurements at autopsy } \\
\text { - US examination time was very short } \\
(5 \mathrm{~min})\end{array}$ & {$[3]$} \\
\hline $\begin{array}{l}\text { Metastatic tumor xenografts } \\
\text { Immunodeficient mice } \\
\text { (NOD-SCID) }\end{array}$ & $\begin{array}{l}\text { - Image-guided injections } \\
\text { - Tumor localization } \\
\text { and sizing }\end{array}$ & $\begin{array}{l}\text { - To establish orthotopic xenograft models } \\
\text { by ultrasound-guided injections into the } \\
\text { adrenal gland and kidney (subcapsular) } \\
\text { - Tumor location, size, and relation to the } \\
\text { adrenal gland and kidney were } \\
\text { monitored accurately and weekly in US } \\
\text { - Technique is efficient, with up to } 10 \text { mice } \\
\text { injected per } 2 \text {-h session } \\
\text { - Mice had fast recovery times of less than } \\
15 \text { min without procedure-related } \\
\text { morbidity or mortality }\end{array}$ & {$[10]$} \\
\hline $\begin{array}{l}\text { Hydronephrosis/congenital } \\
\text { obstructive nephropathy } \\
\text { (megabladder (mgb) } \\
\text { mouse) }\end{array}$ & Evaluate hydronephrosis & $\begin{array}{l}\text { - Total renal length (RL) and renal pelvic } \\
\text { anterior-posterior diameter (APD) were } \\
\text { measured. Hydronephrosis was scored } \\
\text { by the ratio of APD to RL } \\
\text { - US permits easy stratification of mice into } \\
\text { consistent pathological study groups } \\
\text { composed of mild, moderate, and } \\
\text { severe kidneys. This approach } \\
\text { significantly improves the utility of this } \\
\text { animal model }\end{array}$ & [14] \\
\hline
\end{tabular}


Table 1

(continued)

\begin{tabular}{|c|c|c|c|}
\hline Disease (animal model) & Usage of US and PA & Findings and Remarks & References \\
\hline $\begin{array}{l}\text { Hydronephrosis and } \\
\text { underlying etiologies in } \\
\text { (aging mice) }\end{array}$ & $\begin{array}{l}\text { - Detection of } \\
\text { hydronephrosis } \\
\text { - Identification of concurrent } \\
\text { abdominal lesions }\end{array}$ & $\begin{array}{l}\text { - Underlying etiologies included } \\
\text { pyelonephritis secondary to a preputial } \\
\text { gland abscess and ascending urinary } \\
\text { tract infections; cystolithiasis, infiltrative } \\
\text { abdominal neoplasia with invasion of } \\
\text { the renal pelvis, hilum, and parenchyma } \\
\text { and surrounding lymph nodes; mouse } \\
\text { urologic syndrome, and a case of } \\
\text { spontaneous hydronephrosis with } \\
\text { ureteritis }\end{array}$ & [6] \\
\hline $\begin{array}{l}\text { Acute kidney ischemia } \\
\text { (induced by unilateral } \\
\text { clamping in mice) }\end{array}$ & $\begin{array}{l}\text { - Oxygen } \\
\text { saturation measurements }\end{array}$ & $\begin{array}{l}\text { - Longitudinal measurement of oxygen } \\
\text { saturation shows differences between } \\
\text { mild and severe renal injury } \\
\text { - Kidney volume was also determined } \\
\text { - PA provided a sensitive measure of } \\
\text { change in } \mathrm{sO}_{2} \text { in the acute phase of AKI, } \\
\text { with the } \mathrm{sO}_{2} \text { at } 24 \mathrm{~h} \text { after } \mathrm{AKI} \text { being } \\
\text { predictive of renal function in the } \\
\text { chronic phase }\end{array}$ & {$[15]$} \\
\hline $\begin{array}{l}\text { Acute kidney injury } \\
\text { (bilateral renal ischemia } \\
\text { induced by clamping in } \\
\text { mice) }\end{array}$ & $\begin{array}{l}\text { - Renal microperfusion } \\
\text { imaging }\end{array}$ & $\begin{array}{l}\text { - Contrast-enhanced ultrasonography with } \\
\text { high-resolution parametric perfusion } \\
\text { maps can monitor changes in renal } \\
\text { microvascular perfusion in space and } \\
\text { time in mice }\end{array}$ & [16] \\
\hline $\begin{array}{l}\text { Acute renal ischemia } \\
\text { induced by systemic } \\
\text { hypoperfusion } \\
\text { (Sprague-Dawley rats) }\end{array}$ & $\begin{array}{l}\text { - Study hemorrhagic shock } \\
\text { changes with } \\
\text { microimaging }\end{array}$ & $\begin{array}{l}\text { - After } 1 \mathrm{~h} \text { of ischemia, B-mode images } \\
\text { documented slight changes in kidney } \\
\text { echogenicity } \\
\text { - Color and PW-Doppler analysis showed a } \\
\text { reduction in renal blood flow in kidneys } \\
\text { during the hypoperfusion with a } \\
\text { progressive and significant change from } \\
\text { baseline values of resistive index }\end{array}$ & [17] \\
\hline $\begin{array}{l}\text { Vesicoureteral reflux } \\
\text { (C57BL6/J and } \mathrm{C} 3 \mathrm{H} / \mathrm{HeJ} \\
\text { inbred mice) }\end{array}$ & $\begin{array}{l}\text { Monitor reflux with US } \\
\text { contrast }\end{array}$ & $\begin{array}{l}\text { - Reflux was detected in the proximal } \\
\text { ureters and the renal pelvis bilaterally in } \\
\mathrm{C} 3 \mathrm{H} / \mathrm{HeJ} \text { mice } \\
\text { - Contrast-enhanced ultrasound } \\
\text { cystograms were established in-vivo }\end{array}$ & [18] \\
\hline BALB mice & $\begin{array}{l}\text { Contrast agent } \\
\text { biodistribution in US and } \\
\text { photoacoustic imaging }\end{array}$ & $\begin{array}{l}\text { - A dual imaging contrast agent was } \\
\text { developed to monitor kidney perfusion } \\
\text { and organ distribution as well as whole- } \\
\text { body biodistribution }\end{array}$ & [9] \\
\hline $\begin{array}{l}\text { Chronic kidney disease and } \\
\text { cardiovascular disease } \\
\text { (induced in C } 57 \mathrm{BL} / 6 \mathrm{~J} \text { mice } \\
\text { by } 5 / 6 \text { nephrectomy) }\end{array}$ & $\begin{array}{l}\text { Assess cardiac dimensions and } \\
\text { function }\end{array}$ & $\begin{array}{l}\text { - Cardiac dysfunction and dilatation of the } \\
\text { left ventricle was observed } 8 \text { weeks after } \\
\text { partial nephrectomy } \\
\text { - A treatment reversed cardiac dilatation } \\
\text { and significantly improved cardiac } \\
\text { dysfunction }\end{array}$ & [19] \\
\hline
\end{tabular}




\subsection{Evaluating Kidney Size and Anatomy}

B-Mode, as introduced in Measurement concepts (2.), is used to locate the kidneys in the abdomen, observe the surrounding tissue and anatomical structure and to obtain organ size.

When viewed using ultrasound, the kidneys are located lateral of the abdominal aorta. The right kidney is positioned slightly more cranial in the thoracic cavity then the left kidney, and both can be identified by their characteristic appearance in ultrasound: the medulla in the medial portion of the kidney is darker than the cortex and surrounding the cortex, the kidney capsule appears as a bright thin line. Once the appearance of kidneys and surrounding in ultrasound are familiar, it is easy to identify any changes: Cysts are liquid-filled structures and therefore are visible as black, round structures with in the tissue. Hydronephrosis is qualitatively recognized as distension and dilatation of the renal pelvis and a method to quantify hydronephrosis based on the proportion of renal parenchyma in a longitudinal ultrasound image as been established [5] and underlying etiologies have been studied [6]. Tumor masses will appear in a different grayscale than the surrounding tissue and when becoming larger will distort the regular bean shaped form of the kidney. The observation and sizing of the kidney in 2D images can be performed in the transverse (Fig. la, b) and sagittal imaging (Fig. lc, d) plane as required, based on preference. Moving through the whole organ in in both imaging planes in $2 \mathrm{D}$ allows to systematically screen for changes inside and also in the surroundings (e.g., ascites in the peritoneum). In addition, ultrasound imaging also may be used to study cardiovascular disease linked to chronic kidney disease [19].

Most preclinical ultrasound systems also allow to gather threedimensional information. The principle for $3 \mathrm{D}$ image acquisition is similar between ultrasound and MRI where both acquire sequential 2D images with a known distance between them, determining the resolution along the $z$-axis (slice thickness). In ultrasound imaging a motor needs to be attached to move the probe across the area of interest along the $z$-axis and acquire a stack of images (Fig. 3). The consecutive $2 \mathrm{D}$ slices can be compiled into a $3 \mathrm{D}$ view, displayed in various ways, and analyzed to obtain accurate volume information of the kidneys and also cysts or tumor masses within.

When ultrasound waves are reflected by moving blood a frequency shift (Doppler shift) of the return ultrasound signal is induced that allows to display and measure blood flow. Most ultrasound systems are equipped with several imaging modes that use this Doppler effect to display blood flow in 2D images and allow for measuring blood flow velocities.

Color Doppler mode, overlaid on top of a B-Mode image for anatomical reference, displays blood flow in the area of interest in gradients of red and blue (Fig. 4b). The color gradients indicate both the direction and mean velocity of the Doppler shift: blood 


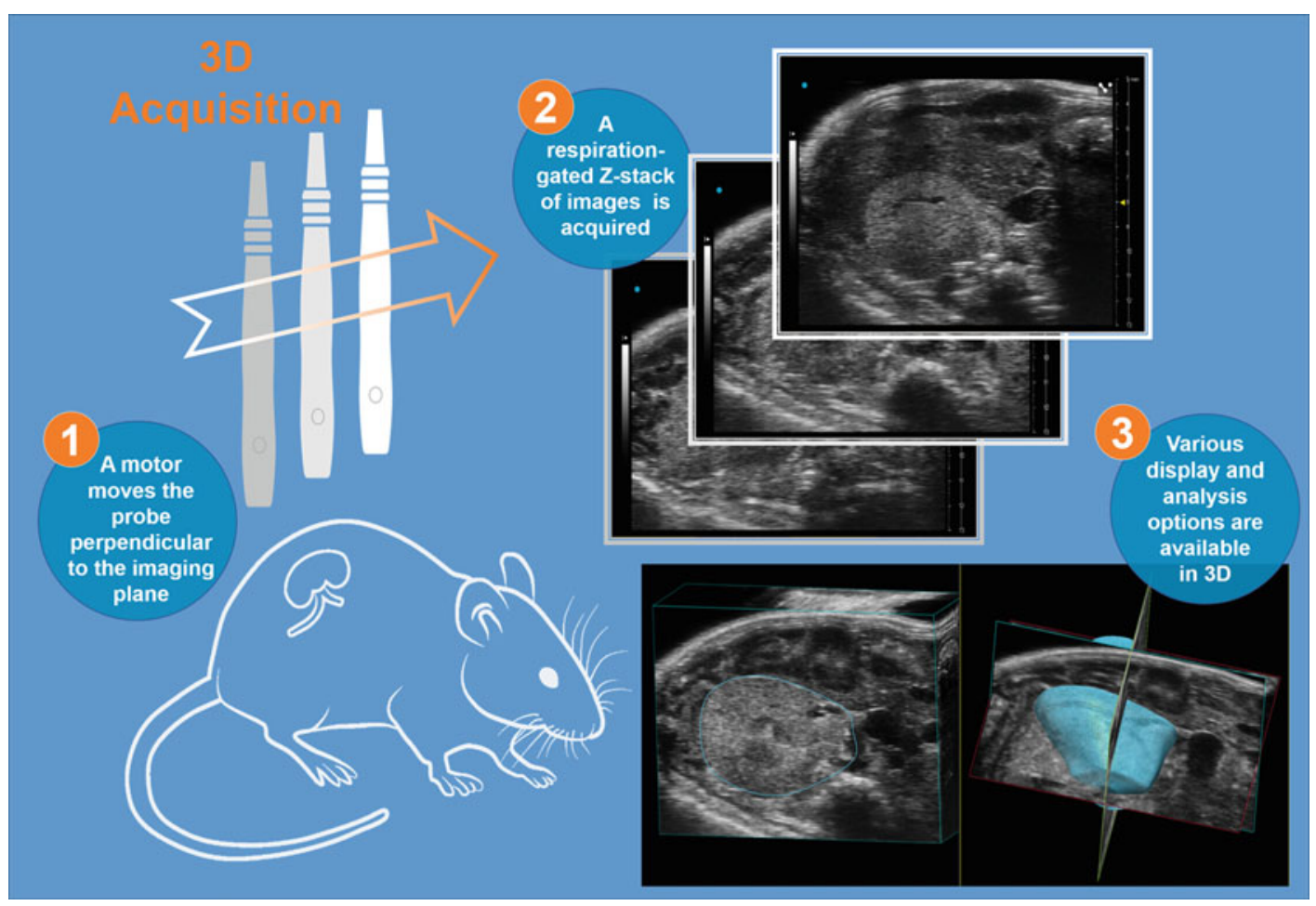

Fig. 3 Schematic workflow for 3D US image acquisition and processing

flowing toward the transducer is represented by a gradient between red (lowest velocity) and white (highest velocity) and blood flowing away from the transducer is represented by a gradient between blue (lowest velocity) and white (highest velocity). BART is an acronym commonly used to remember the colors associated with the direction of flow: Blue Away, Red Toward. Color Doppler mode is a very useful tool to identify the major and cortical vessels in the kidney and accurately guide the positioning for flow measurements, which are performed in pulsed-wave (PW) Doppler mode. To obtain a measurement, a Doppler sample volume (point where the blood flow should be measured) is placed within the vessel and the system generates a PW Doppler spectrum that displays time on the $x$-axis and flow velocity on the $y$-axis (Fig. 4d). Once PW Doppler spectrums of the renal artery and vein have been recorded, velocity measurements such as peak systolic velocity (PSV), end-diastolic velocity (EDV), and the velocity time integral (VTI) can be added. Based on these measurements, relevant parameters will be calculated as an indicator for vascular function.

- The resistive index (RI) is an example of additional analysis that may be useful. A RI of 0 is calculated for continuous flow patterns and an RI of 1 indicates a strongly pulsating flow with no flow during diastole. In the clinic an $\mathrm{RI}$ of the renal 


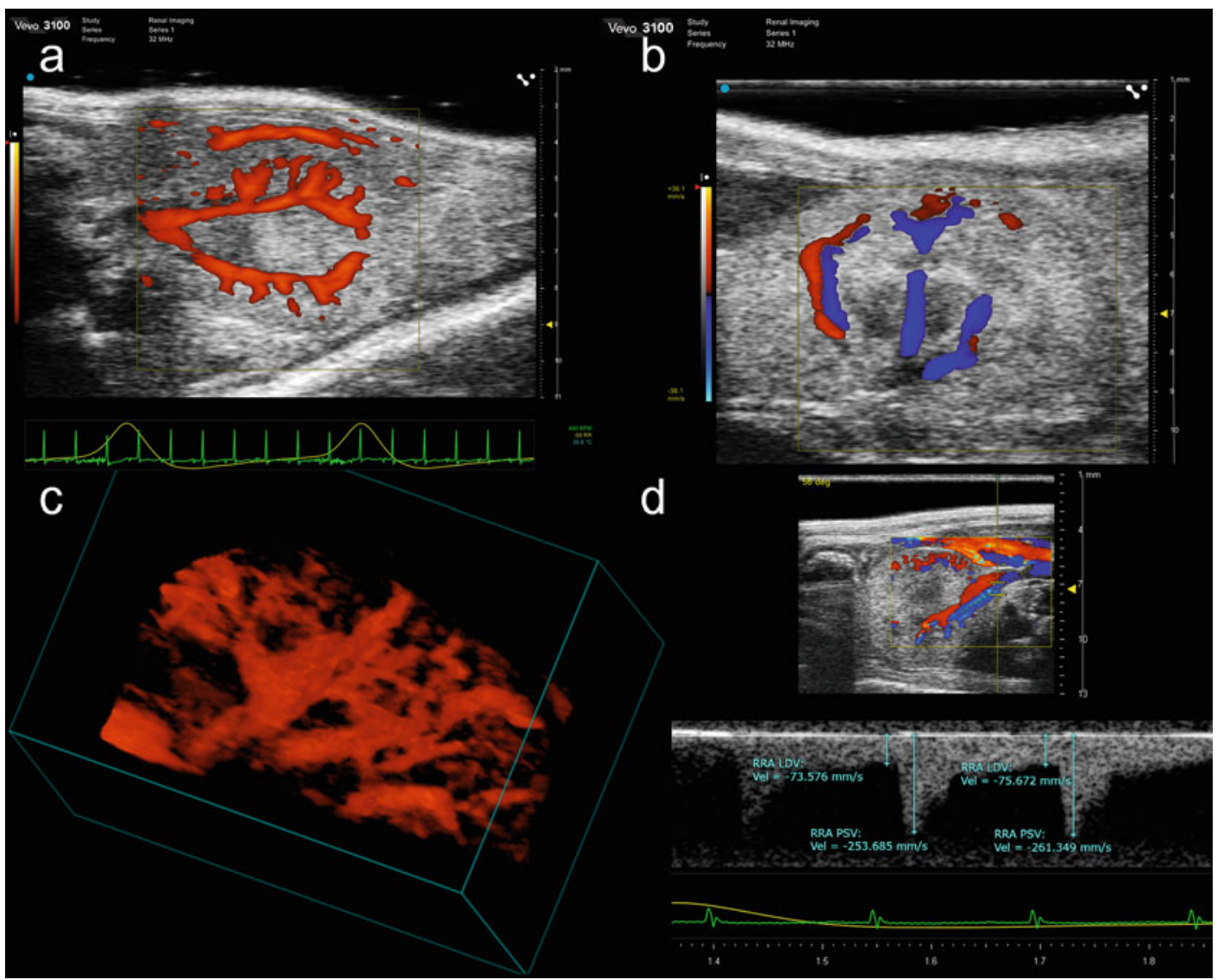

Fig. 4 Assessment of blood flow; (a) power Doppler signal in transverse view of the left kidney is overlaid with the B-mode image, (b) Blood flow in the interlobal vessels as visualized in Color Doppler Mode, in this image red and blue colors indicate arterial and venous flow, respectively; (c) three-dimensional rendering of Power Doppler signal of a healthy kidney, (d) pulse-waved Doppler profile of renal artery flow, peak systolic (PSV), and lowest diastolic velocity (LDV) measurements are shown, the sample positioning is indicated in the scout image above the flow profile

artery $=0.6$ is observed in healthy adults and a rise in $\mathrm{RI}$ is seen as an indicator for renal disease.

$$
\mathrm{RI}=\frac{\mathrm{PSV}-\mathrm{EDV}}{\mathrm{PSV}}
$$

- The pulsatility index (PI) is another parameter often calculated in clinical renal diagnosis.

$$
\mathrm{PI}=\frac{\mathrm{PSV}-\mathrm{EDV}}{\text { VTI mean velocity }}
$$

Power Doppler mode is another tool used to display and analyze blood flow within an area of interest. It is similar to color Doppler mode in detecting the occurring Doppler shift, but instead 
of displaying blood flow direction, it color-codes flow from orange to yellow according to signal intensity and is most useful for smaller vessels having slower blood flow (Fig. 4a).

It is crucial for PW Doppler mode and all other Doppler imaging modes, that an appropriate angle is achieved between the transmitted ultrasound beam and the blood flowing through the vessel; the imaging plane should be adjusted such that the angle between these two is less than $60^{\circ}$ to provide an accurate velocity measurement.

Color and power Doppler information can also be acquired in $3 \mathrm{D}$, visualizing the blood vessel structure in the entire organ (Fig. 4c). The amount of Doppler signal in a 3D volume can then be calculated. With the right animal setup, imaging both kidneys at the same time is feasible.

\subsection{Perfusion Imaging}

\subsection{Molecular Information}

While Doppler imaging can be used to visualize blood flow in the renal artery and vein and also in the interlobar and arcuate vessels, monitoring perfusion of the kidney at capillary level requires the use of ultrasound contrast agents. Ultrasound contrast agents are very small gas bubbles (microbubbles) about $2-3 \mu \mathrm{m}$ in diameter and encapsulated by a polymer or lipid shell (Fig. 5a). Several contrast imaging modes can be used for the detection of these microbubbles in the area of interest. Linear contrast enhanced ultrasound, the first contrast mode used at frequencies above $15 \mathrm{MHz}$, primarily relies on subtraction of background information to display the increase in echo due to the wash-in of contrast agent. Newer generation systems allow to monitor perfusion in real-time based on nonlinear fundamental detection with amplitude modulation, resulting in a higher sensitivity [7].

While ultrasound imaging to monitor perfusion requires a contrast agent and therefore intravenous access, photoacoustic imaging allows for the detection of hemoglobin and its oxygen saturation status (Fig. 2). Based on the change of hemoglobin's optical properties dependent on oxygen bound to the heme group, one can assess tissue oxygenation at a level matching BOLD (blood oxygen level-dependent) MRI [8]. It should be noted that ultrasound contrast agent and photoacoustic imaging of hemoglobin are nontoxic and harmless to the animal.

Ultrasound and photoacoustic contrast agents can also be used for molecular imaging. Ultrasound contrast agents can be targeted to assess biomarker expression (Fig. 5c) but are exclusively tailored to vascular endothelium as the contrast agent does not leave the vasculature. Photoacoustic contrast agents, dyes or nanometersized particles, can be used for specifically targeting extravascular targets or for assessing glomeruli function and to study biodistribution [9]. 


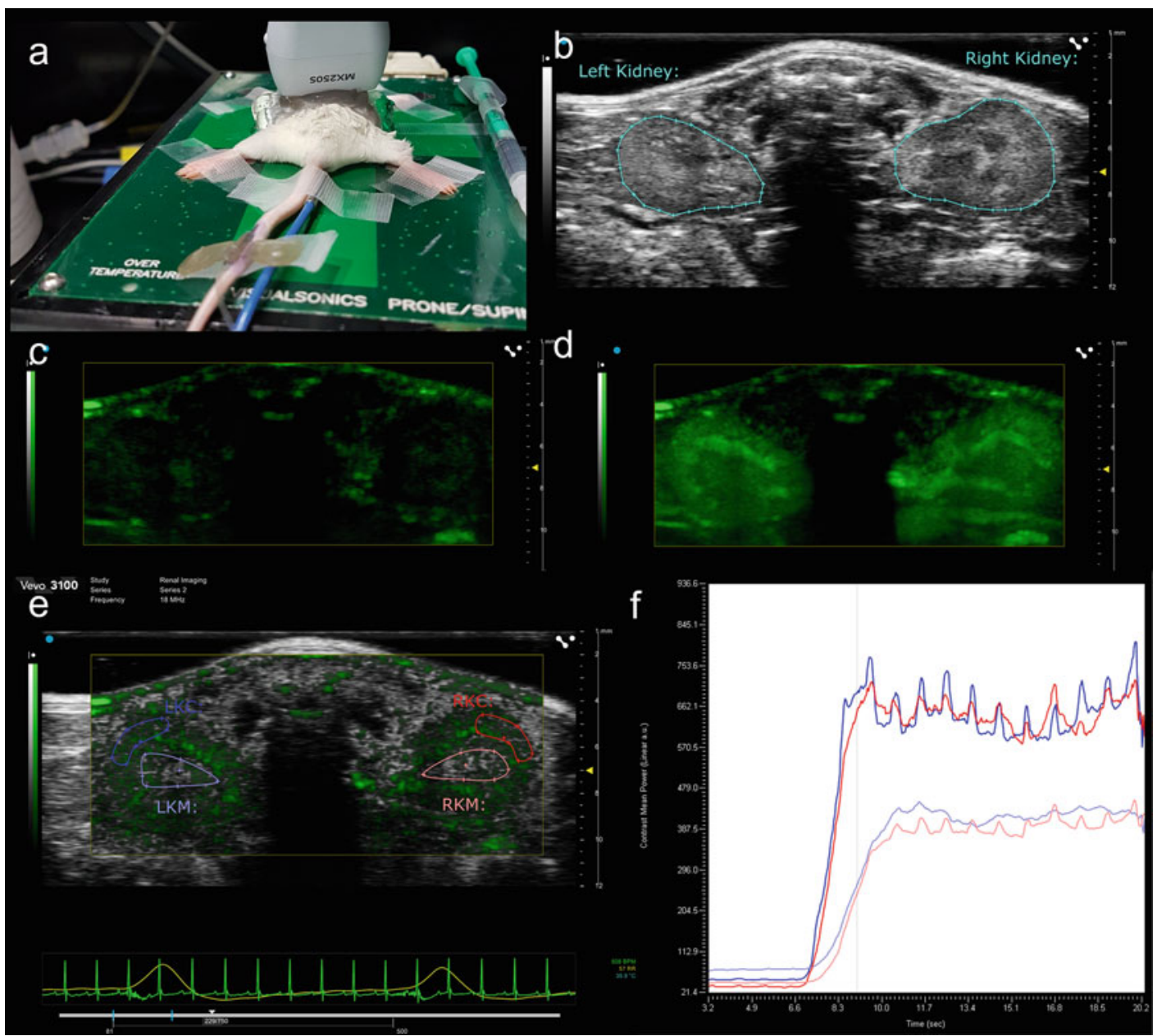

Fig. 5 Perfusion assessment of both mouse kidneys; (a) schematic model of a microbubble used for US contrast imaging; (b) animal and transducer position, (c) B-mode image, left and right kidney are outlined; (d) schematic binding of targeted contrast agent to a biomarker; Non-linear contrast image of both kidneys before (e) and after contrast injection (f). After contrast injection; for analysis, regions of interest in cortex and medulla of left (blue) and right (red) kidney are selected (g); a graph displays the contrast signal in these regions of interest (h)

\subsection{Interventional Procedures}

In contrast to other imaging modalities, in ultrasound and photoacoustic imaging the animal is accessible throughout the imaging session, allowing for various interventional procedures to be performed during an imaging session (Fig. 6). Using a so-called in-plane needle visualization technique, it is possible to follow the movement of a needle inside the animal in B-mode and ensure accurate placement of the needle tip for injections or aspiration. Image guided injections allow to place cells to create orthotopic tumor models or apply drug treatments precisely without the need for more invasive surgery [10]. Switching to image guided injections is an important step in the refinement of animal procedures and helps to shorten recovery time and minimize discomfort and 


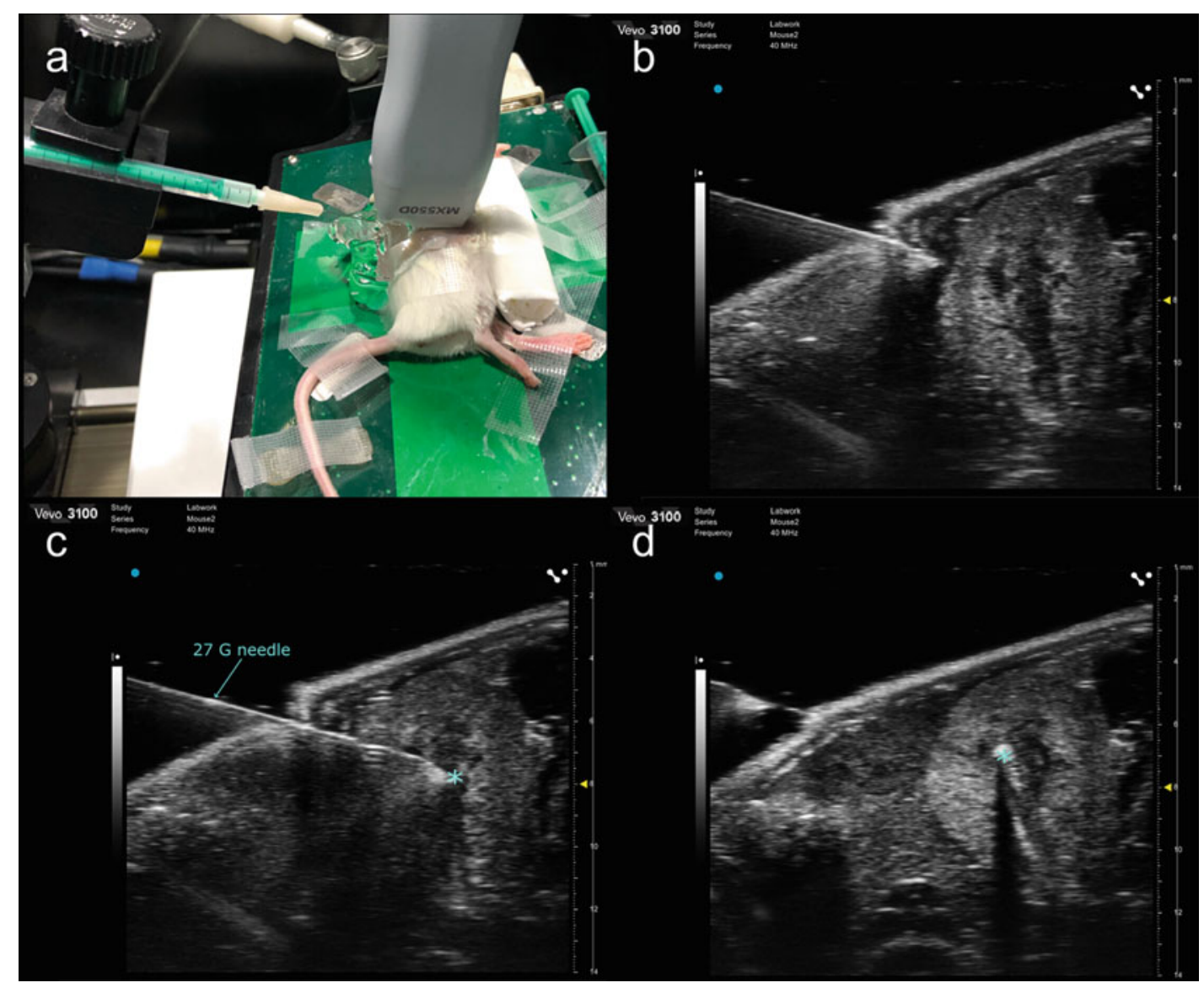

Fig. 6 Image guided injection into the left kidney of a mouse; (a) animal and needle positioning, (b) penetration of the skin, (c) the needle tip (marked with an asterisk) is positioned in the medulla of the kidney, (d) after injection of US contrast agent and needle retraction, injection site is marked with an asterisk

complications as the procedure can be performed with only light anesthesia and the procedure itself does not require postoperative analgesia. In addition the success of any given injection or aspiration can be verified in real time.

\section{Protocols}

\subsection{Standard} Renal Exam
The protocol outlined below depicts a standard renal ultrasound exam that can be performed on various ultrasound systems. If available, a system dedicated for small animal imaging using highfrequency linear transducers is be preferred due its superior resolution and sensitivity. For rats a transducer with a 50 or $75 \mu \mathrm{m}$ resolution is appropriate and for mice a transducer with $40 \mu \mathrm{m}$ resolution should be used. Despite the similar principle of various ultrasound systems, the availability of imaging modes, settings and 
analysis tools may vary. Please refer to instructions provided by the manufacturer for system-specific information and tailored work protocols.

While some brief ultrasound scans (e.g., for linear measurements, screening for pathologies or anatomical alterations) can be performed on awake animals, more comprehensive renal exams including flow measurements or volumetric analysis normally require the use of anesthesia. A brief description of isoflurane anesthesia is included below, but researchers should always carefully follow local rules and regulations.

In order to ensure reproducibility and to streamline workflow in longitudinal studies, it is recommended to store settings for all imaging modes and applications, stick to the same anesthesia type and level, as well as monitor the animal's physiologic parameters.

1. Switch on the flow of oxygen or medical air (the latter preferred for contrast enhanced ultrasound imaging) and set it to $1 \mathrm{~L} /$ min, place the animal in the induction box and turn on isoflurane at $\mathbf{4 - 5 \%}$ until anesthesia is induced. Once a sufficient level of anesthesia is reached (loss of righting reflex), transfer the animal onto the imaging table and reduce isoflurane to 1.5-2\% during imaging (depending on the animal model), or higher for interventional procedures. Make sure to switch the flow of anesthesia gas from the knock out box to the mask.

2. Place the animal in supine position if the renal exam is part of an abdominal scan; a prone position may be used to evaluate kidney structure, dimensions and volume, this position is also more suitable for image-guided injections.

3. Prepare the animal: apply eye ointment, tape the paws to the ECG leads using a small amount of ECG gel for coupling, insert a rectal probe to monitor body temperature and remove the hair in the area of interest. Animal physiology should be carefully monitored throughout the imaging session and adjustments made as necessary; for example, an additional heat lamp can be used to maintain body temperature.

4. Remove hair from the area of interest using a dedicated clipper and/or hair removal cream.

5. Apply a sufficient amount of prewarmed ultrasound gel, place the transducer in transverse position below the ribcage and start imaging in B-mode. Check the transducer orientation. In a supine position the imaging convention needs to be followed similar to MRI imaging, that is, with a transverse imaging plane the head should be behind the image, tail toward the viewer, animal's left on the right side of the screen.

6. Locate the area of interest by moving from the lower end of the ribcage toward the lower abdomen. The right kidney can be localized caudally of and adjacent to the liver. The left kidney is 
located caudally of the stomach and in close proximity to spleen and the tail of the pancreas (Fig. la).

7. The image can be optimized by adjusting the field of view to fit the kidney, focus and gain. Localize the widest, middle part of the kidney and center the organ on the screen using the micromanipulators of the animal station. When imaging in a supine position, the animal table may be tilted sideways to bring the kidney closer to the transducer and remove shadowing caused by the intestinal tract. Save a B-Mode video clip of the transverse view for analysis (Fig. Ib).

8. Switch on color Doppler to display the blood flow in the renal artery and vein. Adjust the Doppler window to fit the position and size of the organ and fine-tune the transducer position to locate the vessels. Save a video clip, then activate pulsed-wave Doppler mode to measure blood flow in the renal artery by placing the Sample Volume gate within the artery. Align the angle of the PW Doppler with the direction of blood flow as indicated by color Doppler mode - you may need to reposition the transducer to obtain an angle smaller than $60^{\circ}$. Velocity, gain and baseline may be adjusted to display the flow profile and should be saved as presets in longitudinal studies. Save the blood flow profile for analysis (Fig. 4d).

9. With the transverse view centered on the screen, the sagittal view of the kidney can be displayed by rotating the transducer $90^{\circ}$. Confirm the longest extension of the kidney is displayed by a mid-lateral movement and save a B-Mode video clip of the sagittal view for analysis (Fig. ld).

10. If required, intrarenal flow in the interlobar arteries can also be displayed and measured. Starting from the sagittal view, the transducer should be adjusted to the coronal plane. Fine-tune the positioning until the vessel structure (in B-Mode) and flow pattern (in color Doppler mode, Fig. 4b)) can be visualized, then switch on pulse-waved Doppler to record the flow pattern.

11. In addition a $3 \mathrm{D}$ data set of the area of interest can be obtained either in the transverse or sagittal view. Most commonly a 3D motor is used to move the transducer perpendicular to the imaging plane to acquire a stack of images (Fig. 3). Select a start and end position or scanning range and start 3D acquisition. Respiration gating may be applied to avoid movement artifacts due to the breathing of the animal. Once completed the data set can be processed to display a 3D view of the organ and saved for analysis.

12. A combined 3D acquisition of B-Mode and power Doppler or color Doppler may be used to also obtain blood flow information for the whole organ (Fig. 4c). In this case, power or color 


\subsection{Perfusion Studies}

Doppler should be activated before starting the $3 \mathrm{D}$ acquisition and the size of the window should be enlarged to cover the region of interest, the use of respiration gating and a wall filter is recommended to avoid respiration artifacts.

13. Confirm all images required for analysis have been saved. Wipe off the ultrasound gel, turn off the isoflurane and release the animal from the mouse handling table. Monitor the animal until fully recovered.

Studying kidney perfusion in ultrasound imaging requires the intravenous injection of a contrast agent. Administration can be performed either as a continuous infusion using a pump, or as bolus injection, which can be delivered by hand but using a pump is recommended to ensure reproducibility. In addition it is possible to study reperfusion by applying a short ultrasound pulse to destroy the contrast agent within the field of view and record subsequent reperfusion. Here we would like to outline a basic perfusion study that can be included in an imaging session in combination with the standard exam. To allow for comparison, the imaging procedure should be standardized as much as possible. For a detailed review of contrast imaging in small animals and various parameters that should be considered, please refer to [11].

1. Before anesthetizing the animal, follow the instructions provided by the manufacturer to reconstitute the US contrast agent. Prepare a tail vein catheter (butterfly needle suitable for the contrast agent, fine tubing connected to syringe filled with saline).

2. Start animal anesthesia as described above. Once the animal has been moved to the animal table and eye ointment has been applied, insert the saline filled butterfly needle into the tail vein and fix the catheter with tape or tissue glue. Position and prepare the animal for imaging to obtain anatomical and blood flow information as described above using the 22-55 MHz probe (see Subheading 4.1).

3. Switch ultrasound transducer if required for high sensitivity detection (e.g., 15-24 MHz transducer for 2-3 $\mu \mathrm{m}$ phospholipid shell bubbles). Contrast imaging for one kidney can be performed in various positions, if perfusion of both kidneys should be observed at the same time, a prone position may be used (Fig. 5c, d). Position the animal as required and apply a small amount of gel. Avoid air bubbles as these may cause artifacts.

4. Start the nonlinear contrast (NLC) mode, adjust the imaging window, and adjust the gain so that a small amount of background signal is visible in the area of interest (Fig. 5e). Check the frame rate of the image acquisition and adjust the video clip 
length to allow for sufficient acquisition time (e.g., $30 \mathrm{~s}$ ), locate the prospective saving option on the system. If required, acquire a $3 \mathrm{D}$ data set before contrast injection and do not move the animal or transducer. Once the settings have been optimized it is advised to store the settings as presets.

5. Fill a new syringe with contrast agent, flush the catheter with $15 \mu \mathrm{L}$ of saline and change to the syringe containing the contrast agent. Start the video clip with automatic storage option. After $3 \mathrm{~s}$ inject the bolus of $50 \mu \mathrm{L}$ of contrast agent within $3 \mathrm{~s}$ using a pump for accurate injection rates. Within a few seconds a signal increase in the area of interest should be visible and the video clip will be stored automatically (Fig. 5f).

6. Change back to the syringe containing saline and flush the catheter with $15 \mu \mathrm{L}$ and if required, record a second 3D data set using the previous settings. Circulating contrast agent will be observed for approximately $10-15 \mathrm{~min}$. Once the contrast agent has been cleared a second bolus injection can be performed if required. After completing the contrast acquisition end the exam (see Subheading 4.1) or continue with one of the options described below.

4.3 Photoacoustic Exam to Monitor Oxygen Saturation
In addition to $2 \mathrm{D}$ and $3 \mathrm{D}$ ultrasound imaging showing renal anatomy, blood flow, and perfusion, photoacoustic (PA) imaging can add additional information by visualizing endogenous as well as exogenous contrast agents.

1. Anesthesia and animal preparation for PA imaging are identical to the procedure described above, but in order to obtain the best possible PA images the animal should be placed either in a lateral decubitus position or prone position to image both kidneys. In a prone position white gauze may be placed underneath the flanks of the animal to ensure reproducible positioning as well as optimizing light delivery.

2. Depending on the system, choose the appropriate photoacoustic transducer or the ultrasound transducer with laser fiber combination. Apply enough gel to the recess of the transducer so that it is overflowing and ensure that no bubbles are present.

3. Apply a generous amount of prewarmed, bubble-free gel to the animal. Lower the transducer into the gel just until contact is made and an image appears in B-Mode. Position the animal or transducer such that the skin line appears horizontal and at the recommended distance from the transducer. This ensures optimized and reproducible light delivery into the tissue, resulting in accurate photoacoustic data.

4. To limit the field of view to the target, adjust image depth, depth offset, and image width. If required, record ultrasound images in B-Mode or Doppler modes as described above. 


\subsection{Image Guided Needle Injections}

5. Switch from B-mode to PA-mode, and start imaging at a single wavelength (e.g., $800 \mathrm{~nm}$ ). First, increase the PA gain to determine if there is any signal present that may be missed with lower gain. Adjust the gain so that low-level signal can still be seen while keeping background signal (often referred to as "noise") to a minimum.

6. To compensate for light attenuation at depth, adjust the time gain compensation or fluence correction. Try to "even out" the signal throughout the depth of the image. For example, the signal at the surface of the skin can be quenched while applying a slight boost with increasing depth. All settings should be saved and reapplied in future imaging sessions.

7. Switch to oxygen detection and record a video clip for Oxygen Saturation analysis, a 3D data set may also be obtained.

8. If required, also obtain a spectral scan or a multiwavelength scans in 2D and 3D based on previously established protocols for imaging exogenous contrast agents such as dyes (e.g., Evans Blue) or nanoparticles.

9. End the exam as described above or continue to perform an interventional procedure.

Image guided interventions can be added to any imaging session as an additional step to locally administer a drug or implement cells. This procedure can be performed independently and is a fast and reliable procedure to replace a surgical intervention. Please refer to Subheading 4.1 for instructions on anesthesia, animal preparation, and imaging and follow these additional steps:

1. An analgesic treatment may be applied before the start of the procedure.

2. Prepare a syringe for injection and attach a $30 \mathrm{G} \mathrm{l}$ in. needle. If the use of a different needle is required, please ensure the length of the shaft is sufficient for the needle to pass under the probe without the needle hub pressing against the probe casing. Fix the syringe in an injection mount to allow for precise control of the needle movement directly under the transducer, for best visualization of the needle tip the bevel should be facing upward.

3. If available, an additional micromanipulator can be placed between transducer and transducer mount, this allows to finetune the probe position for clear identification of the needle tip during the procedure.

4. Adjust the positioning of the needle and transducer. The needle should be aligned with and parallel to the ultrasound beam, which can be checked by placing the needle in some ultrasound gel and lowering the transducer into it (Fig. 6c). When 
positioned correctly, the whole length and tip of the needle should be visible on the live image as a bright line. Once a good alignment has been achieved, movement of the needle and probe in the $\mathrm{Y}$-axis should be avoided.

5. The animal may be taped to the electrodes in a prone position or in a lateral decubitus position (Fig. 6a) to inject into the contralateral kidney. In this case, taping the lower front and hind paw and tail to the electrodes will allow to monitor the animal's physiology.

6. Apply a generous amount of bubble-free gel to the area of interest. Lower transducer into the gel and bring the kidney into view. Adjust the position to have some space on the left for needle positioning.

7. If desired, set the ultrasound system up to record a long video clip by adjusting the length of the video and enable prospective saving, start recording before inserting the needle or injection as required.

8. Advance the needle forward into the gel until it is visible. By tilting and moving it up or down, the projected needle path should point to the target area within the kidney. If large blood vessels or critical organs lie within the needle pathway, reposition the animal to avoid unnecessary damage of tissue.

9. Advance the needle quickly to push through the skin, then gently move forward to place the needle tip in the target structure (Fig. 6b). If the needle slightly bends out of view during the injection, the $\Upsilon$-axis micromanipulator can be used to readjust the image. Perform the injection and wait shortly to allow for distribution then slowly retract the needle (Fig. 6d). If desired, save a video clip of the procedure.

10. End the ultrasound exam as described above and monitor the recovery of the animal. Check the animal for signs of pain or complications in regular intervals as indicated in the animal protocol (i.e., up to $24 \mathrm{~h}$ after injection).

\subsection{Analysis}

All commercially available ultrasound systems dedicated to in vivo imaging provide measurement tools for comprehensive image analysis that cover a broad range of standard applications. As data is mostly stored in proprietary file formats, analysis tools are a fundamental part of the system capabilities and convenient to use, no other third party or open source software is normally used for standard analysis. Therefore no detailed step-by-step instructions are given below. Instead the type of measurements routinely performed are outlined below and were applicable, calculations are mentioned. Depending on the ultrasound system that is used, measurements will either be added directly during the exam, afterward on the ultrasound system itself or on a dedicated offline 
analysis software. Please refer to the user instructions to ensure all data required for analysis is saved. Modern preclinical ultrasound and photoacoustic equipment allow for extracting the image data as DICOM or raw formats, making the data accessible in nonproprietary image analysis programs.

1. The length of the kidney in a sagittal and transverse 2D $\mathrm{B}$-Mode images can be measured in $\mathrm{mm}$ using a line measurement.

2. In standard clinical exams, kidney volume is often calculated using the ellipsoid formula: Length $\times$ Width $\times$ Depth $\times 0.523$. Using a 3D motor and appropriate settings a more accurate volume of the kidneys can be obtained. Depending on the software, various ways of volume reconstructions are available. Mostly the user is required to outline the organ in a few, selected frames on either the video clip or reconstructed 3D view. Based on this information an outline of the organ is created and the volume calculated. In addition to the total kidney volume, sub-volumes can be added to measure cysts, tumors or other anatomical structures. Most software packages also allow to copy volume reconstructions between imaging modes and will quantify additional parameters. In 3D Power and Color Doppler images, percent vascularity can be measured in the volume of interest. Various display options are available to visualize results.

3. In PA imaging, the PA signal intensity can be measured within an area of interest at a single wavelength and build in algorithms allow to also calculate oxygen saturation and hemoglobin content in $2 \mathrm{D}$ and $3 \mathrm{D}$. If required, signal from endogenous or exogenous contrast agents can be separated from background using automated spectral unmixing tools.

4. Flow analysis of the renal artery or intrarenal arteries routinely include measuring peak systolic (PSV) and lowest diastolic (LDV) as well as the velocity-time-integral. If not automated in the analysis software, the Resistive Index can be calculated manually based on the first two parameters: (PSV - LDV)/ PSV.

5. A basic perfusion analysis includes outlining areas of interest, for example in the kidney cortex, medulla or a tumor, to display the wash in curve of the contrast agent. Based on this curve, the time to peak, a relative measure for blood flow, and peak enhancement, a relative measure for blood volume, can be measured. If $3 \mathrm{D}$ data is recorded, it is also possible to observe the contrast signal before and after contrast injection and calculate the parameter percent agent.

6. For presentation purposes, ultrasound and photoacoustic imaging analysis software allows for annotations and color 
coding of anatomical structures and further editing for educational purposes or publishing.

\section{Acknowledgments}

This chapter is based upon work from COST Action PARENCHIMA, supported by European Cooperation in Science and Technology (COST). COST (www.cost.eu) is a funding agency for research and innovation networks. COST Actions help connect research initiatives across Europe and enable scientists to enrich their ideas by sharing them with their peers. This boosts their research, career, and innovation.

PARENCHIMA (renalmri.org) is a community-driven Action in the COST program of the European Union, which unites more than 200 experts in renal MRI from 30 countries with the aim to improve the reproducibility and standardization of renal MRI biomarkers.

\section{References}

1. Hansen K, Nielsen M, Ewertsen C (2015) Ultrasonography of the kidney: a pictorial review. Diagnostics 6:2

2. Foster FS, Hossack J, Adamson SL (2011) Micro-ultrasound for preclinical imaging. Interface Focus 1:576-601

3. Linxweiler J, Körbel C, Müller A et al (2017) Experimental imaging in orthotopic renal cell carcinoma xenograft models: comparative evaluation of high-resolution 3D ultrasonography, in-vivo micro-CT and 9.4T MRI. Sci Rep 7:1-10

4. Needles A, Heinmiller A, Sun J et al (2013) Development and initial application of a fully integrated photoacoustic micro-ultrasound system. IEEE Trans Ultrason Ferroelectr Freq Control 60:888-897

5. Carpenter AR, Becknell B, Ingraham SE, McHugh KM (2012) Ultrasound imaging of the murine kidney. In: Michos O (ed) Kidney development. Methods in molecular biology, vol 886. Humana Press, Totowa, NJ

6. Springer DA, Allen M, Hoffman Vet al (2014) Investigation and identification of etiologies involved in the development of acquired hydronephrosis in aged laboratory mice with the use of high-frequency ultrasound imaging. Pathobiol Aging Age Relat Dis 4:1-14

7. Needles A, Arditi M, Rognin NG et al (2010) Nonlinear contrast imaging with an arraybased micro-ultrasound system. Ultrasound Med Biol 36:2097-2106
8. Rich LJ, Seshadri M (2015) Photoacoustic imaging of vascular hemodynamics: validation with blood oxygenation level-dependent MR imaging. Radiology 275:110-118

9. Toumia Y, Cerroni B, Trochet P et al (2018) Performances of a pristine graphenemicrobubble hybrid construct as dual imaging contrast agent and assessment of its biodistribution by photoacoustic imaging. Part Part Syst Charact 35:1800066

10. Noord RAVAN, Thomas T, Krook $M$ et al (2017) Tissue-directed implantation using ultrasound visualization for development of biologically relevant metastatic tumor xenografts. In Vivo 791:779-791

11. Hyvelin J, Tardy I, Arbogast C et al (2013) Use of ultrasound contrast agent microbubbles in preclinical research. Investig Radiol 48:1-14

12. Kapoor S, Rodriguez D, Mitchell $\mathrm{K}$ et al (2016) High resolution ultrasonography for assessment of renal cysts in the PCK rat model of autosomal recessive polycystic kidney disease. Kidney Blood Press Res 41:186-196

13. Westergren HU, Grönros J, Heinonen SE et al (2015) Impaired coronary and renal vascular function in spontaneously type 2 diabetic leptin-deficient mice. PLoS One 10:e0130648

14. Ingraham SE, Saha $M$, Carpenter AR et al (2010) Pathogenesis of renal injury in the Megabladder mouse: a genetic model of congenital obstructive nephropathy. Pediatr Res 68:500-507 
15. Okumura K, Matsumoto J, Iwata Y et al (2018) Evaluation of renal oxygen saturation using photoacoustic imaging for the early prediction of chronic renal function in a model of ischemia-induced acute kidney injury. PLoS One 13:e206461

16. Fischer K, Meral FC, Zhang Y et al (2016) High-resolution renal perfusion mapping using contrast-enhanced ultrasonography in ischemia-reperfusion injury monitors changes in renal microperfusion. Kidney Int 89:1388-1398

17. Iacobellis F, Segreto T, Berritto D et al (2018) A rat model of acute kidney injury through systemic hypoperfusion evaluated by microUS, color and PW-Doppler. Radiol Med 124 (5):323-330

18. Paredes J, Sims-Lucas S, Wang $\mathrm{H}$ et al (2011) Assessing vesicoureteral reflux in live inbred mice via ultrasound with a microbubble contrast agent. AJP Ren Physiol 300: F1262-F1265

19. Yoshida A, Kanamori H, Naruse G et al (2019) (pro)renin receptor blockade ameliorates heart failure caused by chronic kidney disease. J Card Fail 25:286-300

Open Access This chapter is licensed under the terms of the Creative Commons Attribution 4.0 International License (http://creativecommons.org/licenses/by/4.0/), which permits use, sharing, adaptation, distribution and reproduction in any medium or format, as long as you give appropriate credit to the original author(s) and the source, provide a link to the Creative Commons license and indicate if changes were made.

The images or other third party material in this chapter are included in the chapter's Creative Commons license, unless indicated otherwise in a credit line to the material. If material is not included in the chapter's Creative Commons license and your intended use is not permitted by statutory regulation or exceeds the permitted use, you will need to obtain permission directly from the copyright holder. 